\title{
Anaphylaxis by antihistamine containing bovine gelatin: the utility of the basophil activation test in the diagnostic work-up
}

\author{
Eleonora Nucera ${ }^{1}$, Amira Colagiovanni ${ }^{2}$, Simona Mezzacappa ${ }^{1}$, Alessandro Buonomo ${ }^{1}$, Arianna Aruanno ${ }^{1}$, \\ Angela Rizzi ${ }^{1}$, Domenico Schiavino ${ }^{1}$
}

${ }^{1}$ Allergy Unit, Università Cattolica del Sacro Cuore, Policlinico A. Gemelli, Rome, Italy ${ }^{2}$ MCAU Cardarelli Hospital, Campobasso, Italy

Adv Dermatol Allergol 2017; XXXIV (3): 279-280 DOI: https://doi.org/10.5114/ada.2017.67851

Gelatin, a collagen protein obtained from cow and pig bones and fish skin is widely used, by the hydrolysis of collagen, in food preparations (sweets, yoghurt, etc.) and as a stabilizer in the pharmaceutical industry. In particular it could be found as an ingredient of drug capsules, plasma expanders, vaccines and plasma substitutes.

Systemic allergic reactions to gelatin-containing vaccines and drugs have been proven by anti-gelatin IgE assay [1-3] and by the Basophil Activation Test (BAT) [4].

Bovine gelatin sensitization is commonly associated with cow milk allergy and beef allergy [5].

We report the case of a 14-year-old patient affected by cow's milk allergy, who experienced lip paraesthesia, generalized itching and dyspnoea with bronchospasm after the ingestion of lyophilised ebastine; the reaction was handled in the emergency room with intramuscular and aerosolized epinephrine, systemic corticosteroids and antihistamines.

The clinical history revealed an asthmatic reaction after polio vaccination and an egg allergy; for this reason the patient did not undergo any other immunization.

Since the information leaflet of ebastine showed the presence of gelatin as an ingredient of the lyophilised drug, we suspected a bovine gelatin hypersensitivity.

We did not perform either the skin prick test with bovine gelatine and ebastine (kindly provided by the drug manufacturer) or the oral challenge test with ebastine because the patient had previously experienced an anaphylactic reaction after the skin prick test with a commercial extract of milk.

Specific IgE (ImmunoCap Phadia, Uppsala, Sweden) to bovine gelatin and beef was detected and we also carried out the basophil activation test with gelatin and ebastine in order to exclude or confirm hypersensitivi- ty to the antihistamine. In our patient, specific IgE for gelatin and beef result was $0.01 \mathrm{kU} / \mathrm{l}$ and $0.10 \mathrm{kU} / \mathrm{l}$, respectively.

Peripheral whole blood samples were collected in heparinized tubes: $100 \mu$ l of whole blood was pre-incubated with $20 \mu \mathrm{l}$ of the basophil stimulation buffer (containing IL-3). Basophils from the patient and from five healthy controls were stimulated with $100 \mu \mathrm{l}$ of the basophil stimulation buffer as negative control (BD Pharmingen) and with $100 \mu \mathrm{l}(10 \mu \mathrm{g} / \mathrm{ml})$ of anti-lgE as positive control (BD Pharmingen). Then we used, as stimulants, $100 \mu \mathrm{l}$ of bovine gelatin contained in the drug and $100 \mu \mathrm{l}$ of ebastine. The degranulation was stopped on ice, the cells were stained with CD63-FITC/CD123-PE/AntiHLADR-PerCP (BD FastImmune) and the samples were lysed, fixed, washed and analyzed within $3 \mathrm{~h}$ on a FACSCanto flow cytometer (BD, Immunocytometry Systems, San Jose, CA, USA). Activated basophils were gated as CD123+/HLADR-/CD63+ and the results of BAT were expressed as a percentage of CD63+ basophils.

The basophil activation test with gelatine and ebastine resulted in no activation of cells in any of the five healthy controls, while a dose-dependent CD63 up-regulation (from $67 \%$ to $87 \%$ ) was observed in the patient after the stimulation with gelatine. No basophil activation was observed after ebastine stimulation.

In the literature, Apostolou et al. [4] reported a sensitivity of $100 \%$ and a specificity of $87.5 \%$ of the basophil activation test for the diagnosis of Gelofusine allergy.

Hypersensitivity to gelatin can be diagnosed by the detection of the specific IgE and skin prick test, however BAT could be an additional test [6] in fact, even if the skin prick test remains "the gold standard" for the diagnosis of a drug hypersensitivity, our case report is an example

Address for correspondence: Dr Eleonora Nucera, Allergy Unit, Università Cattolica del Sacro Cuore, Policlinico “A. Gemelli”, Largo F. Vito, 1-00168 Rome, Italy, phone: +39 0630154965, fax: +39 0630156999, e-mail: eleonora.nucera@rm.unicatt.it Received: 10.02.2016, accepted: 9.07.2016. 
of the utility of the BAT in the diagnostic work-up of a patient who experienced a history of anaphylaxis during the skin prick test with other allergens.

In conclusion, we suggest that BAT should be considered a safe in vitro test especially when in vivo tests are contraindicated. Moreover it is important to underline that the type of gelatin used as ingredients of drugs should be specified in the information leaflet in order to avoid hypersensitivity reactions in potential allergic patients.

\section{Acknowledgments}

Eleonora Nucera, Amira Colagiovanni and Domenico Schiavino - equally contributed.

\section{Conflict of interest}

The authors declare no conflict of interest.

\section{References}

1. Pool V, Braun MM, Kelso JM, et al. Prevalence of anti-gelatin ige antibodies in people with anaphylaxis after measlesmumps rubella vaccine in the United States. Pediatrics 2002; 110: e71.

2. Sakaguchi M, Inouye S. Anaphylaxis to gelatin-containing rectal suppositories. J Allergy Clin Immunol 2001; 108: 1033-4.

3. Żukiewicz-Sobczak WA, Wróblewska P, Adamczuk P, et al. Drugs as important factors causing allergies. Postep Derm Alergol 2015; 32: 388-92.

4. Apostolou E, Deckert K, Puy R, et al. Anaphylaxis to Gelofusine confirmed by in vitro basophil activation test: a case series. Anaesthesia 2006; 61: 264-8.

5. Bogdanovic J, Halsey NA, Wood RA, et al. Bovine and porcine gelatin sensitivity in children sensitized to milk and meat. J Allergy Clin Immunol 2009; 124: 1108-10.

6. Hausmann OV, Gentinetta T, Bridts $\mathrm{CH}$, et al. The basophil activation test in immediate-type drug allergy. Immunol Allergy Clin North Am 2009; 29: 555-66. 